\title{
Kernos
}

Revue internationale et pluridisciplinaire de religion grecque antique

$26 \mid 2013$

Varia

\section{Florence Gherchanoc, L'oikos en fête. Célébrations familiales et sociabilité en Grèce ancienne}

Adeline Grand-Clément

\section{Q OpenEdition \\ Journals}

Édition électronique

URL : http://journals.openedition.org/kernos/2149

DOI : 10.4000/kernos.2149

ISSN : 2034-7871

\section{Éditeur}

Centre international d'étude de la religion grecque antique

Édition imprimée

Date de publication : 10 octobre 2013

Pagination : 398-401

ISSN : 0776-3824

Référence électronique

Adeline Grand-Clément, «Florence Gherchanoc, L'oikos en fête. Célébrations familiales et sociabilité en Grèce ancienne », Kernos [En ligne], 26 | 2013, mis en ligne le 10 octobre 2013, consulté le 02 mars 2021. URL : http://journals.openedition.org/kernos/2149; DOI : https://doi.org/10.4000/kernos.2149

Ce document a été généré automatiquement le 2 mars 2021.

Kernos 


\title{
Florence Gherchanoc, L'oikos en fête. Célébrations familiales et sociabilité en Grèce ancienne
}

\author{
Adeline Grand-Clément
}

\section{RÉFÉRENCE}

Florence Gherchanoc, L'oikos en fête. Célébrations familiales et sociabilité en Grèce ancienne, Paris, Publications de la Sorbonne, 2012. 1 vol. $16 \times 24 \mathrm{~cm}, 265 \mathrm{p}$. (Histoire ancienne et médiévale, 111). ISBN : 978-2-85944-690-1.

1 La sphère domestique est sans aucun doute l'un des lieux privilégiés pour observer et analyser l'imbrication du «public » et du «privé » dans la cité grecque, notamment en matière de pratiques religieuses ${ }^{1}$ : l'ouvrage de Florence Gherchanoc (F.G.) le montre bien. L'oikos y est appréhendé sous l'angle des « fêtes et célébrations » qui rythment la vie de la maisonnée, principalement aux époques archaïque et classique. L'objectif affiché est de faire converger, d'une part, les études récentes sur les pratiques de sociabilité et, d'autre part, celles sur la famille et la parenté2. On mesure alors l'ampleur et la difficulté de la tâche, tant chacune des deux notions envisagées (famille/ sociabilité) renvoie à une réalité complexe et à un champ d'investigation étendu. Le lecteur est alors en droit de s'interroger : comment cerner les contours de la "famille » dont il sera question ici ? Quelles « fêtes » et « célébrations » seront concernées ? L'A. prend soin, dès l'introduction, de rappeler la définition de l'oikos (une demeure/un patrimoine/une communauté humaine) et d'évoquer la constellation sémantique des mots grecs se rapportant à ce que nous nommons «famille» (oikia, genos, anchisteia,...). F.G. souligne que les Grecs ne la conçoivent nullement comme un cercle fermé mais comme un groupe à géométrie variable, dont les membres entretiennent des liens forts de solidarité et de partage, de philia, qui nécessitent d'être périodiquement redéfinis, resserrés, réaffirmés: telle est la principale fonction des pratiques de sociabilité étudiées dans le livre. L'étude prend ainsi en compte le cercle restreint du «noyau 
familial ", constitué de ceux qui partagent le même toit et la même nourriture, mais aussi le groupe des parents et familiers (suggeneis, prosêkontes, epitêdeioi, oikeioi...), ainsi que les cercles d'amis (philoi, hetairoi) qui gravitent autour. Voilà pour la famille et ses proches. En revanche, les contours de ce qui relève de la "sociabilité » restent davantage flous, comme l'admet l'A. dans la conclusion (p. 205). Qu'est-ce qu'une " fête »? Une " célébration »? À quelles catégories antiques appartiennent les « rituels de sociabilité » rassemblés dans le livre ? Si les sacrifices et repas communs occupent une place de choix, l'A. a pris le parti d'ouvrir l'analyse, en intégrant des pratiques assez variées, tel l'examen préliminaire du nouveau-né auxquels procèdent les Anciens, à Sparte (p. 38).

2 Cette difficulté inhérente à la nature et au périmètre de l'étude - et qui fait aussi tout son intérêt et sa richesse - s'accompagne d'un problème, qu'affronte d'emblée l'A., avec lucidité, dans l'introduction : l'état fragmenté et éclaté de la documentation disponible. F.G. a en effet choisi de rassembler toutes les sources littéraires disponibles (d'Homère aux auteurs d'époque romaine): comme ces œuvres ne forment pas un ensemble homogène, elles obligent l'A. à procéder - selon ses propres termes - à un " bricolage " des sources (p. 19). Poèmes épiques, tragédies et comédies, plaidoyers judiciaires, récits d'historiens, traités philosophiques sont ainsi mobilisés tout au long de l'ouvrage pour nourrir l'analyse, en obligeant à naviguer entre le registre mythique et les données historiques. L'A. puise aussi à plusieurs reprises dans la documentation épigraphique, lorsqu'il s'agit par exemple de réfléchir aux relations entre les célébrations familiales et les autorités civiques, ou au rôle que jouent certaines associations religieuses fondées autour du culte d'un ancêtre commun. De fait, la primauté accordée aux sources littéraires a nécessairement orienté et précisé les contours chronologiques et géographiques de l'enquête : les époques archaïque et classique sont ainsi privilégiées, même si la période hellénistique n'est pas oubliée et permet de cerner certaines évolutions; Athènes constitue de loin l'exemple le plus exploité.

3 Les trois premiers chapitres présentent une vue d'ensemble des principaux rites qui accompagnent les moments marquants de la vie familiale: mariages, naissances et décès. De tels événements nécessitent une recomposition du groupe domestique et une redéfinition de ses contours. Ainsi, les différentes célébrations évoquées dans ces chapitres (proteleia, gamos, pannuchides, Amphidromies, dekatê, prothesis, ekphora, perideipnon,...) visent à tisser, renforcer, renouer les liens de solidarité au sein de la communauté domestique. Au-delà de la variété des situations - et en dépit du caractère dispersé des témoignages littéraires, qui livrent parfois des témoignages dissonants -, il apparaît que le temps fort de ces rituels réside dans la participation à un sacrifice et dans le partage d'un repas commun, qui permettent d'inscrire l'événement dans la "mémoire familiale» (p.35) et de s'assurer la protection bienveillante des dieux. Les deux chapitres suivants élargissent l'enquête à d'autres pratiques de sociabilité, en allant des plus «intimes» aux plus «ouvertes». Dans celui intitulé « Entre soi », l'A. s'intéresse au «noyau familial » et à ce qui se joue autour du foyer. F.G. rappelle que l'accueil de nouveaux-nés, des jeunes mariés ou des esclaves s'accompagne de rites d'agrégation qui mobilisent Hestia, et que la vie religieuse au sein de l'oikos s'organise autour des cultes familiaux adressés à Zeus Herkeios et à Zeus Ktêsios. En élargissant l'analyse à la fête des Kronia, qui déborde d'ailleurs le cadre de l'oikos, l'A. affronte la question de la place et du rôle des esclaves au sein de la vie religieuse domestique, sans pouvoir apporter de réponse assurée, tant les situations ont dû varier dans le temps et 
dans l'espace. Parce que l'oikos n'est pas une cellule fermée et repliée sur elle-même, le chapitre suivant est consacré aux "manières d'ouvrir l'espace domestique » : l'accueil des parents, amis, voisins et hôtes de passage s'accompagne de pratiques d'hospitalité et de commensalité qui obéissent à un certain nombre de règles - dont on s'aperçoit qu'elles mettent toujours en jeu les dieux et la piété. On aurait aimé pouvoir approfondir la question de la mise en espace de ces pratiques de sociabilité et des rituels quotidiens : par exemple, où se trouve l'emplacement du foyer domestique ? Des autels ? Comment les membres de l'oikos circulent-ils à l'intérieur de la maison ? Glissant rapidement sur ces questions, l'A. invoque les «silences de l'archéologie » (p. 103).

4 Les chapitres 6 et 7 constituent l'apport le plus original du livre, en prêtant attention à la mise en spectacle qui accompagne réunions et célébrations familiales, et leur confère une dimension publique. L'éclat des fêtes, les biens qui circulent, les cadeaux offerts lors des noces, l'écho des chants et des danses, le faste des processions et des funérailles contribuent à rendre manifeste la légitimité d'un mariage, à annoncer officiellement une naissance ou un décès. La dimension sensorielle - qu'elle soit visuelle, sonore ou olfactive - des rituels familiaux (est-elle d'ailleurs propre aux rituels familiaux?) permet à ceux qui y participent ou en sont les témoins de partager une expérience commune. Mais le spectacle des fêtes vise aussi à réjouir les dieux, comme le souligne l'A. : l'eukosmia est affaire de piété et l'aidôs reste de mise. Les trois derniers chapitres finissent d'élargir l'angle d'approche, en replaçant les fêtes et manifestations familiales au cœur de la vie de la cité. L'A. y aborde des questions variées, liées au rapport entre oikos et polis: le rôle des phratries, le fonctionnement des fondations funéraires familiales (à cos, Théra et Halicarnasse), les stratégies matrimoniales à des fins politiques, la mise en place des réseaux de philia qui innervent la vie civique, les lois et décrets qui règlementent les mariages et les funérailles privées. Ces différents dossiers permettent de replacer les liens tissés au sein de l'oikos au sein des multiples jeux d'appartenance communautaire qui fondent l'unité de la polis. L'analyse révèle ainsi que l'on ne doit pas considérer la famille comme un noyau antérieur et étranger à la cité, et que cette dernière aurait cherché à intégrer a posteriori. Si l'oikos est bien la cellule de base de la société, comme le rappelle Aristote dans la Politique, elle n'a rien de figé ni de «naturel»: elle est le fruit d'une construction culturelle indissociable du développement de la polis. L'A. conclut ainsi à bon droit que l'oikos constitue un « autre lieu du politique » (p. 185).

5 Le cas de la fête des Apatouries (p. 150-152) révèle bien les interactions qui existent entre oikoi et cité : elle permet d'accorder le temps de la vie familiale, qui possède son rythme propre, avec le calendrier civique. En effet, les rituels accomplis à cette occasion dans le cadre des phratries ne concurrencent pas les fêtes de l'oikos, mais viennent les compléter. Autre exemple: dans le chapitre 10, F.G. souligne que la nécessité de légiférer sur certaines célébrations familiales qui investissent partiellement l'espace public s'explique par la volonté de maintenir le bon ordre et l'harmonie au sein de la cité. Les autorités s'emploient à limiter les débordements émotifs, les manifestations ostentatoires excessives, afin de préserver la communauté des sources potentielles de miasma. Mais l'A. affine l'analyse : fixer une durée limitée pour le deuil, en particulier à l'attention des femmes, vise aussi à s'assurer d'une rapide réintégration de ces dernières au sein de la communauté civique, de manière à préserver le bon déroulement des fêtes civiques qui garantissent la pérennité de la polis - on songe notamment aux Thesmophories. On l'aura donc compris, bien qu'il ne 
s'agisse pas de l'argument principal du livre ${ }^{3}$, la question du rapport aux dieux, de la piété et des normes religieuses traverse l'ensemble du volume, car c'est sous le regard et avec l'assentiment des puissances divines que se nouent et se renforcent les liens familiaux.

\section{NOTES}

1. Une série de travaux récents a souligné la nécessité de dépasser la vision traditionnelle d'une dichotomie entre "privé et "public » dans la cité grecque; pour ce qui concerne le domaine religieux, citons le supplément 15 de Kernos, dont certains articles, comme celui de P. Brulé, traitent précisément de la vie religieuse de la famille: V. Dasen, M. Piérart (éd.), Idia kai dèmosia.Les cadres "privés " et «publics » de la religion grecque antique, Liège, 2005 (Kernos, suppl. 15).

2. On peut se reporter à l'abondante bibliographie que fournit l'A. dans l'introduction.

3. L'A. renvoie pour cela notamment à J. Bodel, S.M. Olyan (éd.), Household and Family Religion in Antiquity. The Ancient World: Comparative Histories, Malden, MA/Oxford, 2008.

\section{AUTEURS}

\section{ADELINE GRAND-CLÉMENT}

Université de Toulouse 2 - Le Mirail 